\title{
NURSE-SY-1-1
}

\section{Wound management}

\author{
Hyejin $\mathrm{MOON}^{*}$
}

Department of Nursing, Korea University Guro Hospital, Seoul, Korea

\section{Definition}

The term 'wound' refers to a condition in which the normal skin structure is broken or destroyed, though the severity and depth may vary widely.

\section{Phases of wound healing}

The repair process for acute wounds onvoloves four major phases: hemostasis, inflammation, proliferation (or regeneration) and maturation.

\section{Types of wound closure}

Classification of repair as primary intention, secondary intention, or tertiary intention healing is based on the ideal of primary surgical closure for all wounds.

\section{Acute versus chronic wounds}

Acute Wounds typically are traumatic or surgical in origin. Chronic wounds fail to and proceed normally through the repair process. Chronic wounds frequently are caused by vascular compromise, chronic inflammation, or repetitive insults to the tissue, and they either fail to close in a timely manner or fail to result in durable closure.

\section{General principles in treatment planning}

A comprehensive plan of care should address three areas: correction of etiologic factors, provision of systemic support for healing and topical treatment that creates and maintains an optimal healing environment.

\section{Wound healing: risk factors for nonhealing}

There is usually not one single factor that results in impaired healing. The factors known to impede wound healing can be categorized as intrinsic and extrinsic factors. Intrinsic factors include factors such as advanced age, immune compromise, psychological stress, hereditary skin disorders, and disease states/comorbidities. Extrinsic factors include factors such as infection, malnutrition, insufficient perfusion/oxygenation, smoking, chemotherapy, radiation therapy, and selected medications (e.g., steroids, anticoagulants, angiogenesis inhibitors).

\section{Conclusion}

Effective management of any wound requires an understanding of the normal repair process and the factors that may interfere with normal repair. This understanding provides the foundation for comprehensive assessment of the wound and of the patient and for selection of interventions designed to optimize healing. In addition, it is necessary to identify the risk factors that affect wound healing and create an environment in which multidisciplinary teams can effectively treat wounds. 